\title{
Electromagnetic wave emission during collision between a current sheet and a fast magnetosonic shock associated with coronal mass ejections
}

\author{
T. Haruki, J. I. Sakai, and S. Saito
}

\begin{abstract}
Laboratory for Plasma Astrophysics, Faculty of Engineering, University of Toyama, 3190 Gofuku, Toyama 930-8555, Japan
e-mail: sakaijun@eng.u-toyama.ac.jp
\end{abstract}

Received 12 April 2006 / Accepted 2 May 2006

\begin{abstract}
Aims. We investigate how the emission of electromagnetic waves can be enhanced when a fast magnetosonic shock wave associated with a coronal mass ejection (CME) collides perpendicularly to a coronal streamer with a stable current sheet. Methods. A two-dimensional relativistic and fully electromagnetic Particle-In-Cell (PIC) code is used.

Results. It is shown that the ions in front of the shock can be accelerated by the surfatron acceleration mechanism. This shock compresses the current sheet, resulting in a local electron temperature anisotropy. The electron Bernstein waves are generated by the local electron temperature anisotropy and they are converted into electromagnetic waves (X-mode) through the linear mode conversion due to density inhomogeneity. As a result, the electromagnetic waves are observed in both forward and backward regions of the shock. The simulation results may be applied to the enhancement of electromagnetic wave emissions when a shock wave associated with CMEs collides with a coronal streamer.
\end{abstract}

Key words. plasmas - shock waves - radiation mechanisms: non-thermal - methods: numerical Sun: coronal mass ejections (CMEs)

\section{Introduction}

The shock waves generated by coronal mass ejections (CMEs) are observed in the interplanetary (IP) medium since they accelerate electrons, which can emit type II radio waves. The frequency drift of the observed type II radio bursts is related to the dynamics of the shock waves. Recent observations of IP type II bursts imply that changes in the shock and CMEs dynamics can be caused by interaction with some structures in the IP medium, for examples, collision with another CME, or shock-shock collision (Gopalswamy et al. 2001). Sheeley et al. (2000) showed examples of fast CMEs associated with the deflection of coronal streamers. van der Holst et al. (2002) observed that when the fast CME collides with a helmet streamer, a split in the type II emission occurs. They modeled this CME-streamer interaction by using a 2-D MHD simulation. Mancuso \& Abbo (2004) investigated the observed bifurcation of the radio emission lanes by modelling the interaction of a piston-driven spherical shock with a vertical current sheet located above the active region.

The interaction between a current sheet and a fast magnetosonic wave (or shock) was investigated as a triggering mechanism of sympathetic solar flares (Sakai 1973; Sakai \& Washimi 1982; Sakai 1983; Sakai et al. 1984) and a magnetic reconnection triggered by a supernova (Tamura et al. 2001).

Previous theoretical and simulation models of interaction between a current sheet and a fast magnetosonic shock wave are based on MHD models. To understand physical processes of the emissions of type II radio bursts during the shock-streamer collision, we must take into account plasma kinetic processes by using particle-in-cell (PIC) simulation. Recently Sakai et al. (2005) showed that the electron Bernstein waves or Z-modes generated near the shock front can be converted into extra-ordinary electromagnetic waves (type II radio bursts) through direct linear mode conversion. Sakai et al. (2006) also simulated the shock-shock collision observed by Gapalswamy et al. (2001) and they found that the type II radio bursts can be enhanced after the shockshock collision.

In the present paper we investigate how the emission of electromagnetic waves can be enhanced when a fast magnetosonic shock wave associated with CMEs collides perpendicularly to a coronal streamer with a stable current sheet, by using a two-dimensional relativistic and fully electromagnetic Particle-In-Cell (PIC) code. It is shown that the ions in front of the shock can be accelerated by the surfatron acceleration mechanism. This shock compresses the current sheet, resulting in the local electron temperature anisotropy. Electron Bernstein waves are generated by the local electron temperature anisotropy and they are converted into electromagnetic wave (X-mode) through the linear mode conversion due to density inhomogeneity. The nonlinear excitation process of the electron Bernstein waves in a pinching current sheet was investigated by Haruki \& Sakai (2001). There, the electromagnetic waves are observed in both forward and backward regions of the shock. The simulation results may be applied to the enhancement of electromagnetic wave emission when a shock wave associated with a CME collides with a coronal streamer.

This paper is organized as follows. In Sect. 2 we present our simulation model and in Sect. 3 we show our simulation results. In the final section we summarize our results. 

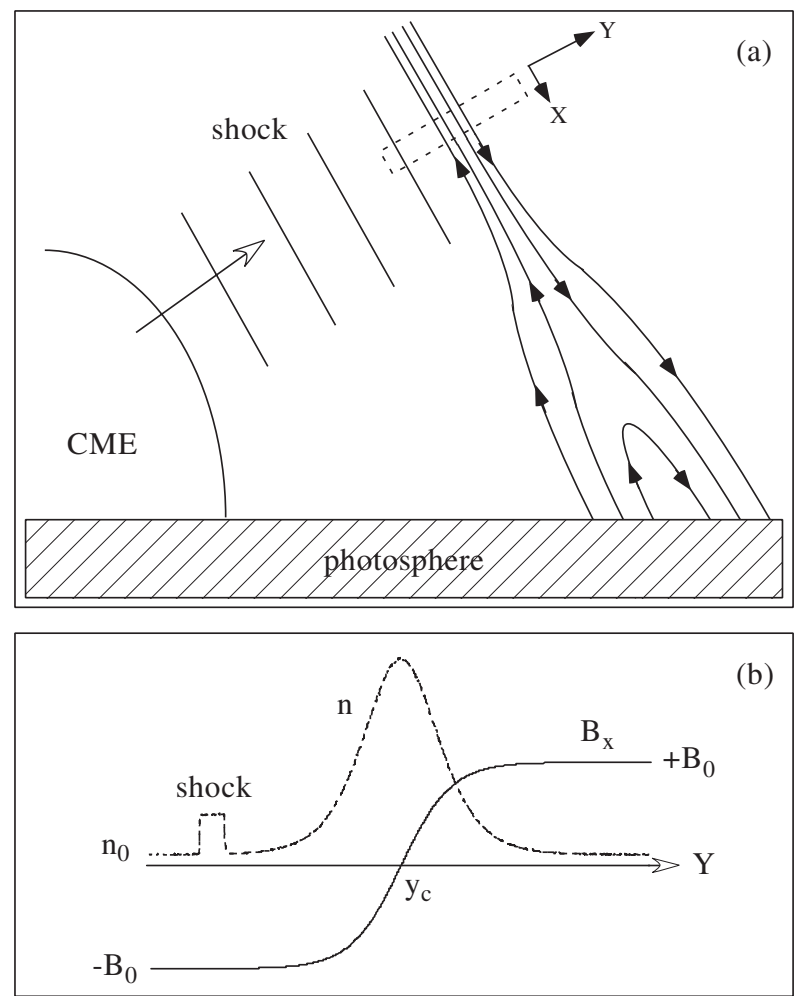

Fig. 1. a) Schematic picture of coronal mass ejection (CME) associated with a shock wave. b) Our one-dimension like simulation model. The solid and dotted lines show the magnetic field and the number density of Harris type current sheet, respectively.

\section{Simulation model}

Figure 1 shows a schematic picture of our simulation model. It is assumed that a CME originating in the lower corona produces a shock, and this shock undergoes a perpendicular collision with a coronal streamer that contains a current sheet as shown in Fig. 1a. Figure 1b displays the initial magnetic field and plasma number density for a Harris type current sheet, corresponding to the dotted box in Fig. 1a. $B_{0}$ is the background magnetic field intensity far from the center of the current sheet, $y_{\mathrm{c}} . n_{0}$ is the background number density, and $n_{\mathrm{c}}$ is the maximum number density at the center of the current sheet. The neutral point of the magnetic field is located at the center of system. On the other hand, we impose a dense plasma cloud far from the center of current sheet to excite a fast magnetosonic shock wave by imposing the plasma velocity with Alfvén Mach speed $M_{\mathrm{A}}=v_{\mathrm{s}} / v_{\mathrm{A}}=3$ on the cloud.

The code used here is a 2-D relativistic and fully electromagnetic PIC code, modified from the 3-D TRISTAN code (Buneman 1993). In order to perform a 1-D-like simulation, the system size in two dimensions is $L_{x}=5 \Delta$ and $L_{y}=2000 \Delta$, where $\Delta(=1)$ is the simulation grid size. It is assumed that the physical quantities are constant in $z$, i.e., $\partial / \partial z=0$. The average number density of background electrons and ions is 100 per cell. For this simulation model, the total particle number is about 3100000 electron-ion pairs. The given particles fill the whole domain. Charge neutrality is initially kept because of same position of electron and ion. Periodic boundary condition in the $x$-direction and free boundary condition in the $y$-direction are imposed on particles and fields.

First, we impose the following Harris type current sheet that is characterized by the magnetic field, current and plasma particle number density, namely,

$$
\begin{aligned}
& B_{x}(y)=B_{0} \tanh \left(\frac{y-y_{\mathrm{c}}}{L}\right) \\
& J_{z}(y)=-\frac{B_{0}}{\mu_{0} L} \cosh ^{-2}\left(\frac{y-y_{\mathrm{c}}}{L}\right), \\
& n(y)=n_{\mathrm{c}} \cosh ^{-2}\left(\frac{y-y_{\mathrm{c}}}{L}\right)
\end{aligned}
$$

where $y_{\mathrm{c}}=1000 \Delta$ and half thickness $L=200 \Delta$. We assume a low beta plasma $\beta=0.1$ and electron thermal velocity $v_{\text {te }} / c=0.1$ ( $c$ is light speed) in the background plasma, and then $n_{\mathrm{c}} / n_{0}=11$ and $\omega_{\mathrm{ce}} / \omega_{\mathrm{pe}}=0.45$ are obtained theoretically, where $\omega_{\text {pe }}$ and $\omega_{\text {ce }}$ are electron plasma and cyclotron frequencies, respectively. Moreover, the Alfvén velocity far from the center of the current sheet is automatically solved with about $v_{\mathrm{A}}=0.045 c$. Next, to excite a shock wave far from the center of the current sheet, we impose a dense plasma cloud whose number density is 200 per cell from $Y=200 \Delta$ to $300 \Delta$. The cloud velocity is set up with $v_{\mathrm{s}}=3 v_{\mathrm{A}}=0.135 \mathrm{c}$.

The other parameters used for this simulation are as follows. The simulation time step is $\omega_{\mathrm{pe}} \Delta t=0.05$, where $\omega_{\mathrm{pe}}$ is used as a normalization of time. The Debye length is $\lambda_{\mathrm{D}}=v_{\mathrm{te}} / \omega_{\mathrm{pe}}=1 \Delta$. The electron skin depth is $c / \omega_{\mathrm{pe}}=10 \Delta$, which is also used as a normalization of space. Mass ratio of electron and ions is $m_{\mathrm{i}} / m_{\mathrm{e}}=100$, whose value is artificial due to computer memory. Temperatures of electrons and ions are the same, $T_{\mathrm{i}} / T_{\mathrm{e}}=$ 1. The Larmor radii of electrons and ions are $2.2 \Delta$ and $22 \Delta$, respectively.

We performed another simulation case without the dense plasma cloud. We confirmed that the current sheet is very stable until $\omega_{\mathrm{pe}} t=1000$. For this case, we did not observe any wave generation.

\section{Simulation results}

Figure 2 shows the development of electron number density in the $y$-direction with time. We found from Fig. 2a that a shock wave is generated by the initial imposed cloud. Figures $2 b-f$ show the propagation of the shock to the center of the current sheet. It is also seen in Figs. 2c-f that the current sheet is compressed strongly by the shock in the region from $Y /\left(c / \omega_{\mathrm{pe}}\right)=50$ to 100 . As more of the shock penetrates to the center of the current sheet, the magnetic field gradually becomes weak, and then the shock propagates slowly. In the phase during which the current is compressed, we confirmed that the electron velocity in the $z$-direction increase twice as much as the initial thermal velocity. Therefore, Harris instability may be expected in this region (Haruki \& Sakai 2001).

Figure 3 shows evidence of ion acceleration through the surfatron acceleration mechanism (Sagdeev \& Shapiro 1973; Sakai \& Ohsawa 1987). Recent observations in IP space (Simnett et al. 2005) confirmed that the surfatron acceleration mechanism is effective for ion acceleration. The electrostatic field $E_{y}$ near the shock front accelerates some ions in the $z$-direction. As seen in Fig. 3a, they are accelerated to almost maximum velocity, $0.2 c$ on $Y /\left(c / \omega_{\mathrm{pe}}=90\right)$. Figure $3 \mathrm{~b}$ shows the ion velocity distribution in the $z$-direction. The above maximum ion velocity is almost the same as the theoretical estimation (Sakai \& Ohsawa 1987).

We investigated the wave emission processes during collision between a fast magnetosonic shock and a current sheet. Figure 4 shows the wave generation of the electron Bernstein 

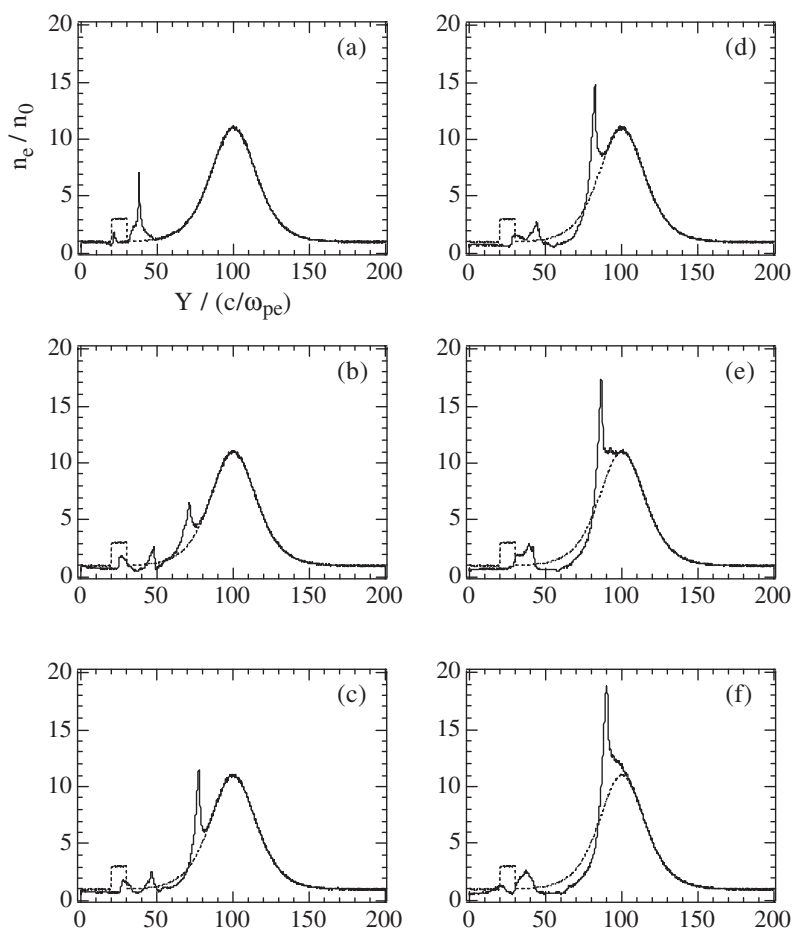

Fig. 2. Time development of electron number density in the $y$-direction at a) $\omega_{\mathrm{pe}} t=100$, b) 400 , c) 500 , d) 600 , e) 700 and f) 800 . The dotted line in all panels displays the initial profile of electron number density. The number density is normalized by the background density $n_{0}$. The space is normalized by electron skin depth $c / \omega_{\text {pe }}$.
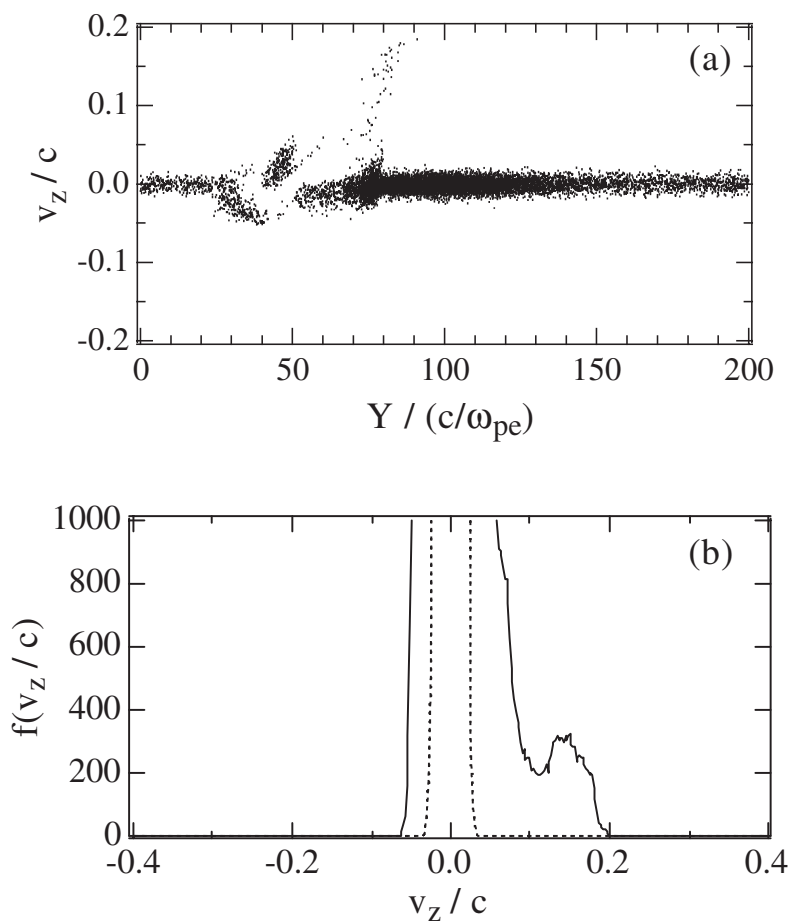

Fig. 3. a) Ion phase snapshot of the velocity component $z$ and space in the $y$-direction at $\omega_{\mathrm{pe}} t=500$. b) Ion velocity distribution in the $z$-direction. A vertical axis is the ion number corresponding own velocity. The velocity in both panels is normalized by light speed.

mode. Figure $4 \mathrm{a}$ shows a line plot of the electric field $E_{y}$ in the $y$-direction at $\omega_{\mathrm{pe}} t=400$. We clearly see the shock front at about $Y /\left(c / \omega_{\mathrm{pe}}\right)=70$. Using the electric field data in the region
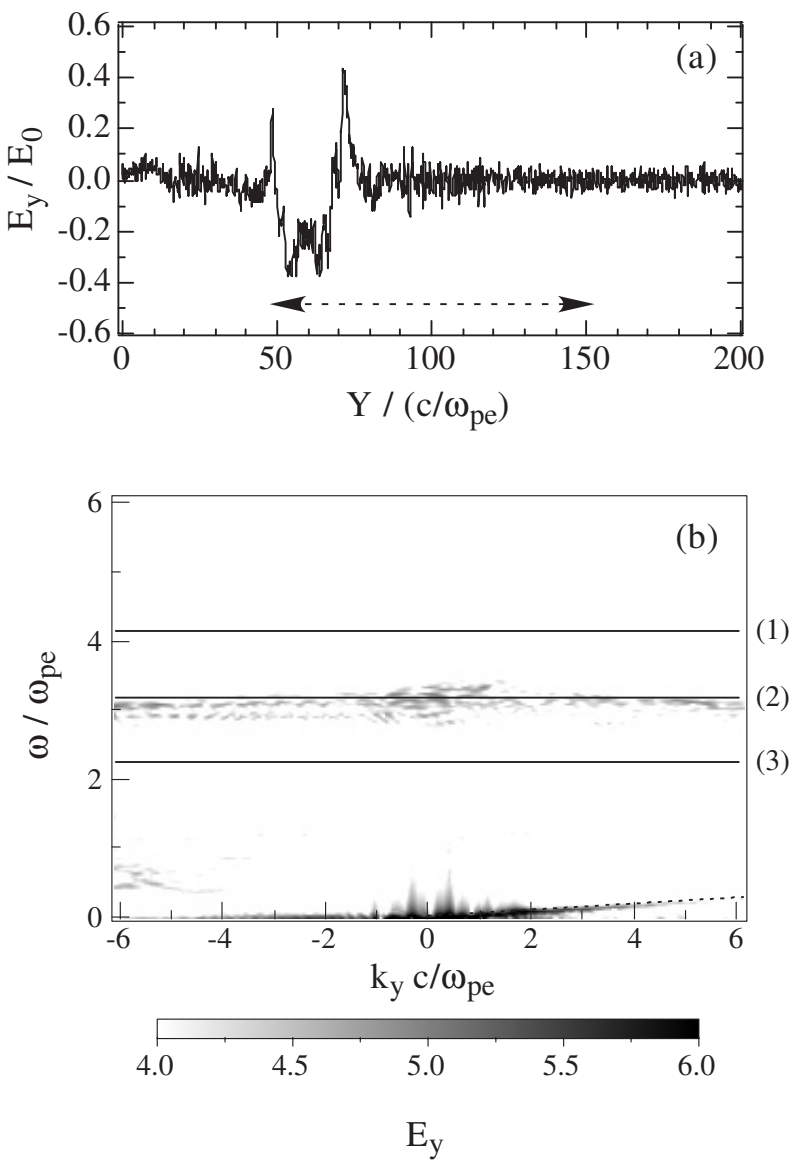

Fig. 4. a) Electric field $E_{y}$ in the $y$-direction at $\omega_{\mathrm{pe}} t=400$, whose intensity is normalized by $E_{0}=m_{\mathrm{e}} v_{\mathrm{te}} \omega_{\mathrm{pe}} / e$. The dotted arrow displays the spatial region for performing Fourier transformation. b) Dispersion relation of $E_{y}$ obtained by space-time Fourier transformation, $48.8 \leq$ $Y /\left(c / \omega_{\text {pe }}\right) \leq 151.2$ and $500.0 \leq \omega_{\text {pe }} t \leq 704.8$. Wave number and frequency are normalized by electron skin depth and electron plasma frequency, respectively. The three solid lines are the theoretical dispersion relation of electron Bernstein mode for (1) $\omega_{\mathrm{ce}} / \omega_{\mathrm{pe}}=4.0$, (2) 3.0 and (3) 2.0. The dotted line of low frequency is the theoretical dispersion relation of magnetosonic mode for $\omega_{\mathrm{ce}} / \omega_{\mathrm{pe}}=0.45$.

indicated by the dotted arrow in Fig. 4a, we performed a spacetime Fourier transformation from $Y /\left(c / \omega_{\text {pe }}\right)=48.8$ to 151.2 $\left(500.0 \leq \omega_{\text {pe }} t \leq 704.8\right)$. We found an electron Bernstein mode in the high frequency range and a magnetosonic mode in the low frequency range. First, we plotted the three theoretical dispersion relations of the electron Bernstein mode for (1) $\omega_{\mathrm{ce}} / \omega_{\mathrm{pe}}=4.0$, (2) 3.0 and (3) 2.0 in Fig. 4b. The theoretical dispersion relation of this mode is obtained from the following equation,

$\frac{2 \omega_{\mathrm{pe}}^{2}}{\omega^{2}-\omega_{\mathrm{ce}}^{2}} \frac{1}{\lambda} I_{1}(\lambda) \mathrm{e}^{-\lambda}=1$,

where $I_{1}$ is the modified Bessel function of the first order and $\lambda=k_{y}^{2} v_{\mathrm{te}}^{2} / \omega_{\mathrm{ce}}^{2}$. We conclude that the generated wave is caused by Harris instability, because temperature anisotropy is observed in this region where the current is compressed. We conclude that the observed low frequency wave is the magnetosonic mode. The peak intensity in this spectrum matches the theoretical dotted dispersion relation well.

Figure 5 shows the time development of the electric field $E_{z}$ in the $y$-direction. From the region of $Y /\left(c / \omega_{\text {pe }}\right)=100$ to 200 in Fig. $5 \mathrm{~b}$, we find that the electric field $E_{z}$ is excited above thermal noise level (Fig. 5a). Figures 5b-c show that the electric 

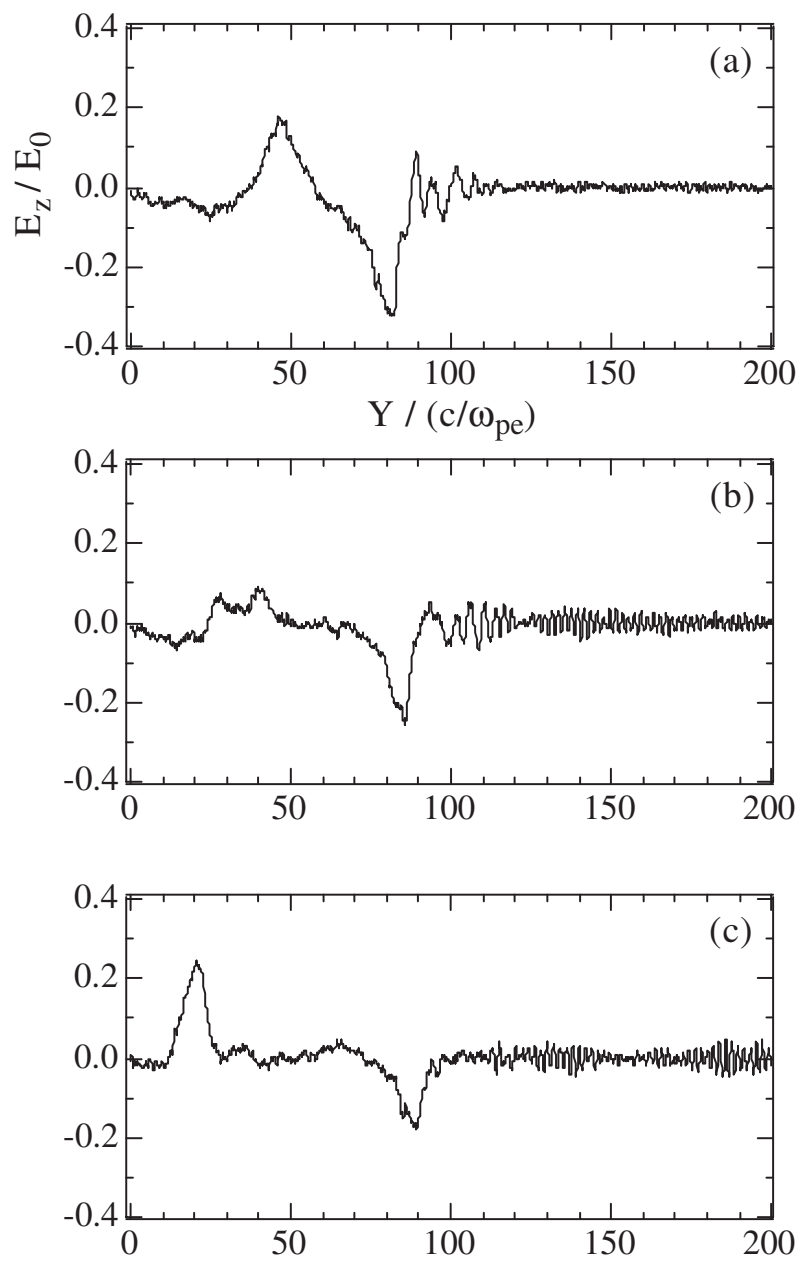

Fig. 5. Time development of electric field $E_{z}$ in the $y$-direction at a) $\omega_{\text {pe }} t=600$, b) 700 and c) 800 .

field perturbation is enhanced by about four times as large as the initial intensity of the electric field that is spontaneously generated from the thermal noise. Compared with Fig. 2, we find that the electric field is enhanced due to the compression of the current sheet driven by the shock wave. To study the characteristics of the excited electric field $E_{z}$, we performed a spacetime Fourier transformation of the $E_{z}$ data. Figure 6 shows forward and backward generation of the X-mode from the current sheet. Both panels show the dispersion relation obtained from the simulation results in the same time period, but the space for the Fourier transformation is different: Fig. 6a is obtained from the data of $120.0 \leq Y /\left(c / \omega_{\text {pe }}\right) \leq 171.2$, while Fig. $6 \mathrm{~b}$ is obtained from the data of $28.8 \leq Y /\left(c / \omega_{\mathrm{pe}}\right) \leq 80.0$. The theoretical dispersion relation of the X-mode for $\omega_{\text {ce }} / \omega_{\text {pe }}=0.45$ is plotted as a dashed curve in both panels. The circle or ellipse in these panels indicates where strong peaks occur. As seen in Fig. 6a, the strong emission of electromagnetic waves occurs along the positive $y$-direction. We conclude that the mechanism for the generation of electromagnetic waves (X-mode) from the current sheet is the linear mode conversion from the electron Bernstein mode due to the density inhomogeneity. The reason for this conclusion is that we find that the high frequency emission with about $3 \omega_{\text {pe }}$ in Fig. 6a agrees well with the result of the electron Bernstein wave in Fig. 4a. On the other hand, we note here that the backward propagating $\mathrm{X}$-mode is also observed as seen in the region marked by the ellipse in Fig. 6 b. (a)

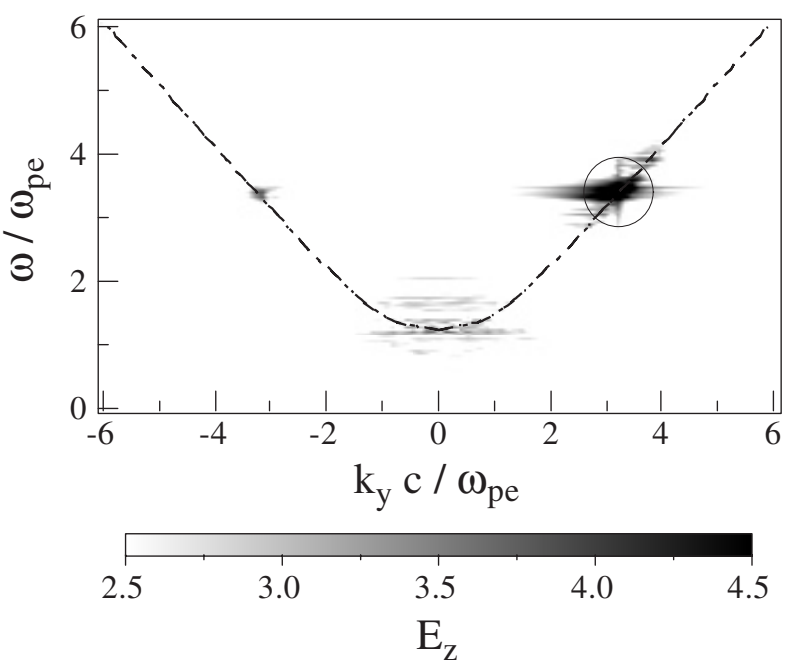

(b)

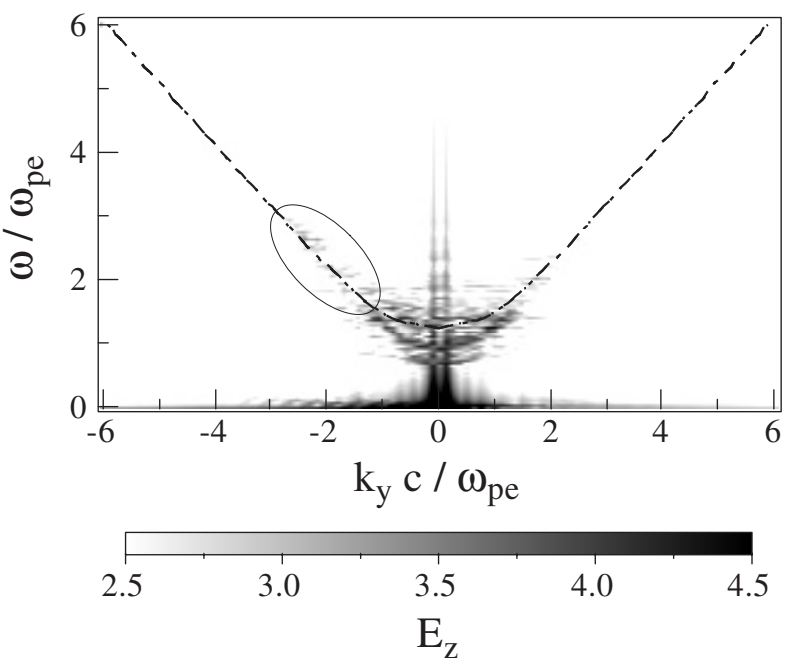

Fig. 6. Dispersion relation of $E_{z}$ obtained by space-time Fourier transformation, a) $120.0 \leq Y /\left(c / \omega_{\mathrm{pe}}\right) \leq 171.2$ and b) $28.8 \leq Y /\left(c / \omega_{\mathrm{pe}}\right) \leq$ 80.0 for $600.0 \leq \omega_{\mathrm{pe}} t \leq 804.8$. The dashed lines in both figures show the theoretical dispersion relation of X-mode for $\omega_{\text {ce }} / \omega_{\mathrm{pe}}=0.45$. The solid circle in (a) and the ellipse in (b) show the region of strong electromagnetic wave emission.

\section{Conclusions}

By using a 2-D relativistic and fully electromagnetic PIC code, we investigated the collision process between a fast magnetosonic shock and a Harris type current sheet. The shock propagates and penetrates into the current sheet. In this propagation period, some ions are accelerated through the surfatron acceleration mechanism in front of the shock wave. The current is compressed by the shock wave and an electron temperature anisotropy appears locally. This electron temperature anisotropy causes the Harris instability. This instability leads to generation of electron Bernstein waves, whose frequency is near $3 \omega_{\text {pe. }}$. The density gap between background plasma and plasma near the center of the current sheet is less than 11. Therefore the frequency of the emitted waves is about the local electron plasma frequency which is proportional to the square root of the local electron number density. The electron Bernstein mode is converted into the $\mathrm{X}$-mode through linear mode conversion due to the density inhomogeneity. Both forward and backward X-mode 
waves propagate perpendicularly to the background magnetic field. Therefore, it is possible to observe the enhancement of the IP type II radio bursts associated with CMEs which collide with a coronal streamer. The two dimensional effect during collision between a current sheet and obliquely propagating shock wave will be studied in future work.

Acknowledgements. The authors thank the anonymous referee for useful comments.

\section{References}

Buneman, O. 1993, in Computer Space Plasma Physics, Simulation Techniques and Software, ed. H. Matsumoto, \& Y. Omura (Tokyo: Terra Scientific), 67 Gapalswamy, N. S., Yashiro, S., Kaiser, M. L., Howard, R. A., \& Bougeret, J.-L. 2001, ApJ, 548, L91
Haruki, T., \& Sakai, J. I. 2001, ApJ, 552, L175

Mancuso, S., \& Abbo, L. 2004, A\&A, 415, L17

Sagdeev, R. Z., \& Shapiro, V. D. 1973, JETP Lett., 17, 279

Sakai, J. I. 1973, Ap\&SS, 23, 285

Sakai, J. I. 1983, J. Plasma Phys., 30, 109

Sakai, J. I., \& Ohsawa, Y. 1987, Space Sci. Rev., 46, 113

Sakai, J. I., \& Washimi, H. 1982, ApJ, 258, 823

Sakai, J. I., Tajima, T., \& Brunel, F. 1984, Sol. Phys., 91, 103

Sakai, J. I., Mori, T., \& Saito, S. 2005, A\&A, 442, 687

Sakai, J. I., Mori, T., Saito, S., Tanaka, Y., \& Aurass, H. 2006, A\&A, 454, 983 Sheeley, N. R., Hakala, W. N., \& Wang, Y. M. 2000, J. Geophys. Res., 105, 5081

Simnett, G. M., Sakai, J. I., \& Forsyth, R. J. 2005, A\&A, 440, 759

Tanuma, S., Yokoyama, T., Kudoh, T., \& Shibata, K. 2001, ApJ, 551, 312

van der Holst, B., van Drel-Gesztelvi, L., \& Poedts, S. 2002, in Solar variability: from core to outer frontiers, ESA SP-506, 1, 71 